\title{
Ionic strength and the supporting material strongly influence the adhesion of silica to supported lipid bilayers
}

Christoph. G. Wittmann ${ }^{l}$, Andrej Kamenac ${ }^{1,2}$, Florian G. Strobl ${ }^{1,2}$, Dietmar Czubak ${ }^{l}$, Achim Wixforth $^{1,2,3}$, and Christoph Westerhausen ${ }^{1,2,3 *}$

1 Chair for Experimental Physics 1, University of Augsburg, 86159 Augsburg, Germany;

2 Nanosystems Initiative Munich (NIM), Schellingstraße 4, 80799 Munich, Germany;

3 Center for NanoScience (CeNS), Ludwig-Maximilians-Universität Munich, 80799 Munich, Germany

C. G. Wittmann, A. Kamenac, F. G. Strobl, D. Czubak, Prof. A. Wixforth

Institut für Physik, Universitätsstraße 1, 86159 Augsburg, Germany

E-mail: christoph.westerhausen@gmail.com

Dr. C. Westerhausen

Institut für Physik, Universitätsstraße 1, 86159 Augsburg, Germany

Keywords: supported lipid membranes, silica nanoparticles, adhesion energy, nanoparticle uptake, giant uni lamellar vesicles

In the context of cell-nanoparticle interaction, the question arises how ions influence the adhesion between nanoparticles and cell membranes. Here, the question is addressed how the adhesion energy between supported lipid bilayers and silica particles help to understand nanoparticle uptake. Supported lipid bilayers on mica and glass substrates serve as simple, well-defined and reproducible membrane models. Using atomic force microscopy with modified cantilevers, the interaction energy of both is found to result in a distinctive dependence on the supporting material. The adhesion energy for 1,2-Dioleoyl-sn-glycero-3phosphocholine on mica substrates shows a clear dependence on the $\mathrm{NaCl}$ concentration. On glass supports, in contrast, stronger adhesive forces, lacking a systematic dependence are found. This is related to experiments where the uptake of silica nanoparticles in free-standing vesicles is observed. While the qualitative dependence of the interaction strength on the ion concentration is definitely confirmed, the quantitative values of the adhesion energy can hardly be transferred to vesicles. These prospects and challenges also hold for investigations of more complex biological cell membranes in which increasing complexity inevitably also 
will increase the challenges. Due to the limited transferability of results obtained in situations of different symmetry and geometry the combination of different methods is suggested.

\section{Introduction}

Since Gorter and Grendel first reported in 1925 that the basic constituent of a cell membrane is a lipid bilayer ${ }^{[1]}$, bilayers are often used as model systems to mimic living cells and cell membranes for biological research ${ }^{[2,3]}$. The most frequently used systems are unilamellar vesicles and supported lipid bilayers (SLB). SLB also play a crucial role in modern bio engineering due to their applications in biosensing applications ${ }^{[4]}$. In many reports, the formation of bilayers on different substrates ${ }^{[5-7]}$ and the resulting quality in terms of continuity and domain formation is examined ${ }^{[8]}$. To understand the internal dynamics of bilayers, multiple studies focus on the diffusion within lipid bilayers ${ }^{[9,10]}$ and the influence of the substrate on bilayer properties ${ }^{[11,12]}$.

On the other hand, vesicle experiments are often employed as a model system to study the interaction of cells and particles. These days, apart from natural sources, also industrially created nanoparticles are widely found in the environment and used as food additives, for drug delivery or as contrast agents in medicine ${ }^{[13,14]}$. These particles can interact in different ways with cell membranes. There are several reports on the penetration of membranes by small nanoparticles ${ }^{[15,16]}$, particles binding to a membrane ${ }^{[17,18]}$ and the uptake of particles by endocytosis-like pathways. In the latter process, three subsequent stages are widely accepted: In short, first, the particles adhere to the membrane, then start to become engulfed by the membrane, until they eventually are fully engulfed and the membrane neck ruptures. The uptake of nanoparticles into lipid vesicles has been a focus of our research group in the past years, motivated by the increasing use of mesoporous silica particles as drug carriers ${ }^{[19]}$. We demonstrated for example the connection between size dependence and in vitro cytotoxicity of silica NPs, the chemical and mechanical impact on the main phase transition of 
phospholipid vesicles ${ }^{[20]}$, as well as the size and membrane state dependency of intake of silica NPs in Giant Unilamellar Vesicles (GUV) ${ }^{[21]}$. Furthermore, presently ongoing studies indicate ion dependent uptake kinetics of silica nanoparticles in GUV (unpublished, see below). In contrast to more complex biological systems these simple membrane model systems can be described by theoretical models offering the chance to derive predictions on physico-chemical effects in particle cell-interactions. Even if biological membranes are drastically reduced in their complexity, pure lipid membrane experiments can help to elucidate important general aspects in biological processes ${ }^{[22]}$.

The adhesion strength between membrane and particle plays an important role for all of the abovementioned models. Therefor the question arises which model systems are suitable for studying adhesion forces. From an experimental point of view, SLB are ideal systems for force-spectroscopic investigations due to their well-defined geometry. However, there is only little information on particle-SLB interaction. For example, the interaction between a micasupported lipid bilayer and silica spheres was investigated earlier by Anderson et al. ${ }^{[23]}$. Their measurements in pure water exhibited a purely repulsive behavior, while in a $150 \mathrm{mM} \mathrm{NaCl}$ PBS solution, strong adhesion was observed. To our best knowledge, there are no other and further reports on systematic studies on the adhesion energy between silica and phospholipid membranes.

Motivated by this and our findings on ion dependent uptake kinetics in vesicles, we here focus on the interaction forces between silica particles and lipid bilayers, being supported either by mica or glass. To allow for AFM measurements we use micro particles for adhesion experiments while nanoparticles are used for all uptake experiments. Moreover, we systematically study these forces in solutions of different $\mathrm{Na}^{+}$ion (i.e. $\mathrm{NaCl}$ ) concentrations. We put our experimental findings in context with existing literature and also discuss the role 
of the bilayer manufacturing process, the supporting material of the SLB as well as the question to what extent SLB represent freestanding lipid membranes in adhesion experiments.

\section{Results \& Discussion}

Our experimental setup is illustrated in Figure 1. In a nutshell, the cantilever with the bead was brought into contact with a supported lipid bilayer and then separated again. The repulsive and attractive forces were then measured for different salt concentrations in the surrounding solution, using the force spectroscopy mode of the AFM. To adjust the ion concentrations, $150 \mathrm{mM}$ HEPES (4-(2-Hydroxyethyl)piperazine-1-ethanesulfonic acid) and $150 \mathrm{mM} \mathrm{NaCl}$ stock solutions were mixed with ultrapure water. All measurements containing salt in the solution were done in the presence of buffer.
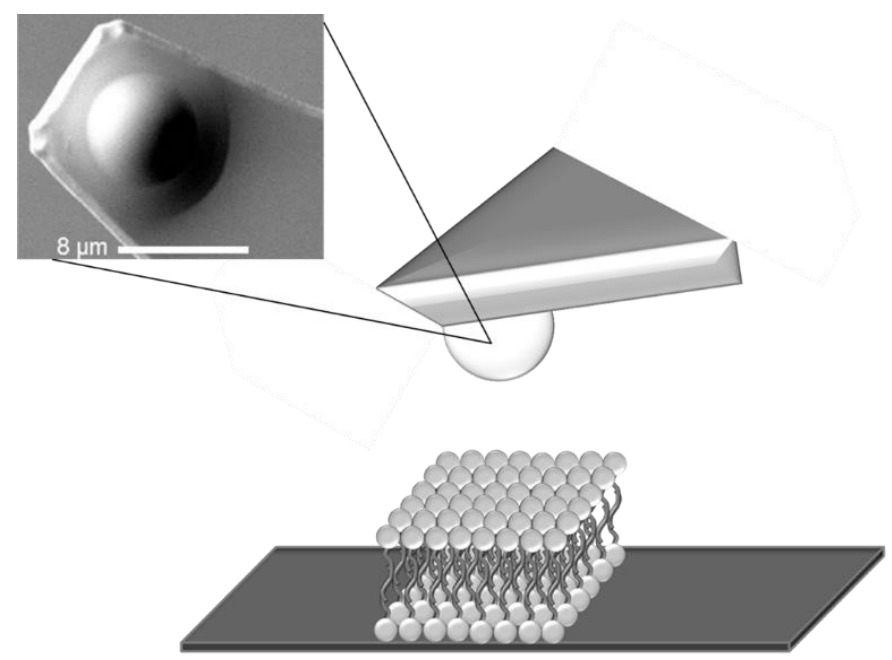

Figure 1. Illustration of the experimental setup. A DOPC bilayer is formed on top of a mica or glass substrate in aqueous solutions of different ionic concentrations. A silica bead, with a diameter of $7.38 \mu \mathrm{m}$, was glued to the cantilever.

Figure 2A shows the three phases of the AFM force spectroscopy measurements. Initially, the silica bead is pushed onto the bilayer up to a distinct force setpoint to ensure proper bead bilayer contact. Next, the height of the silica bead is kept constant for an idle time $t$. Finally, after this idle time, the bead is retracted from the bilayer and the adhesive forces can be determined, before the cantilever eventually detaches at a rupture force $f$. 
A

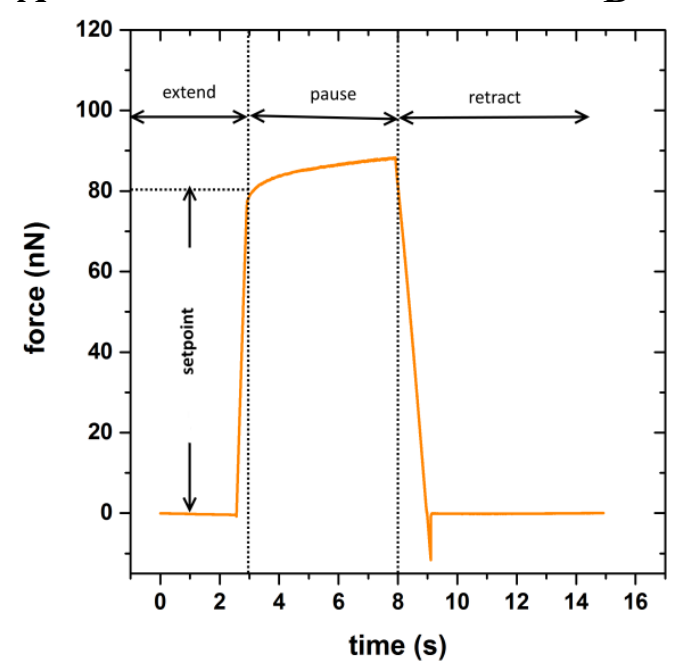

B

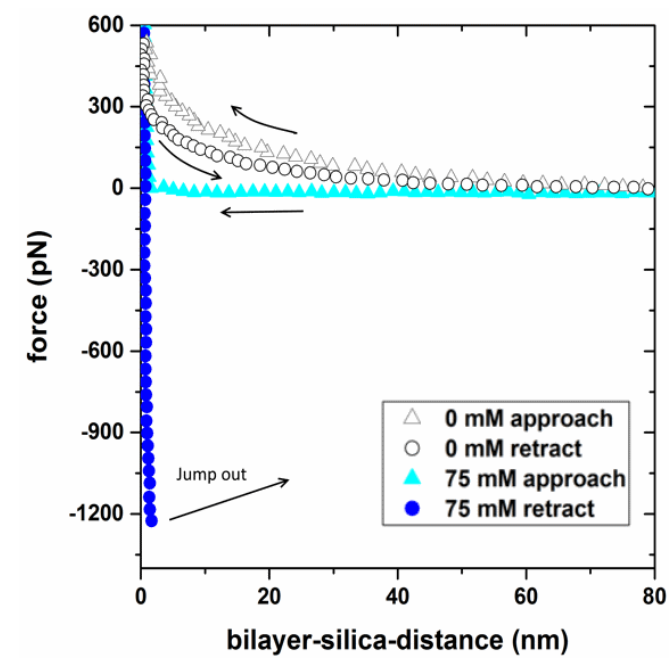

Figure 2. A: Typical force vs. time profile on a glass substrate. The approach speed is 0.5 $\mu \mathrm{m} / \mathrm{s}$ and the setpoint value is $80 \mathrm{nN}$. In the pause segment the constant height mode of the AFM is used. The retraction speed is $0.21 \mu \mathrm{m} / \mathrm{s}$. B: Normalized force-distance curves for bilayer-silica interaction on mica substrate in pure water and in a $75 \mathrm{mM} \mathrm{NaCl}$ HEPES solution. For pure water no adhesion is detected while for the $75 \mathrm{mM}$ solution strong adhesion forces of about $\mathrm{f} \approx 1300 \mathrm{pN}$ can be observed.

Figure 2B shows a set of typical force-distance curves for mica support. The measurements in pure water $(0 \mathrm{mM})$ show purely repulsive behavior and the approach and retraction curves show only slight deviations. In contrast, the measurements in a $75 \mathrm{mM} \mathrm{NaCl}$ HEPES solution clearly indicate adhesive interaction with strongly differing approach and retraction curves. This qualitative behavior is in agreement with an earlier report, where similar measurements were done using a surface force apparatus ${ }^{[23]}$. From these experiments, we can extract the respective rupture forces for DOPC bilayers on mica support and for a variety of different $\mathrm{Na}^{+-}$concentrations. In Figure 3, we depict a set of histograms of those rupture forces for different concentrations in the range of $15 \mathrm{mM}$ and $75 \mathrm{mM}$. All histograms exhibit more or less a Gaussian shape and only one single maximum. With increasing salt concentration, the observed adhesion force increases up to a saturation value of about $1500 \mathrm{pN}$. 


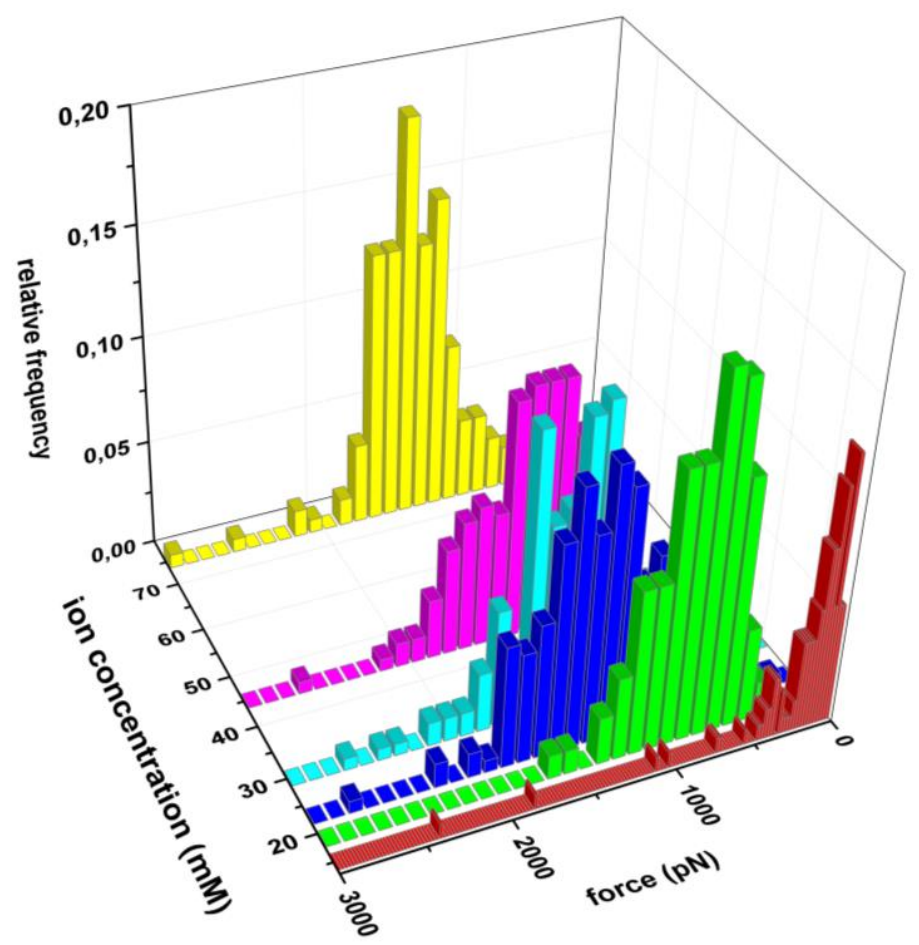

Figure 3. Histograms of adhesion forces for six different ion concentrations on mica. The bins for $\mathrm{I}=15 \mathrm{mM}$ are less wide to account for the smaller distribution width. The evaluated number of force curves ranges between $n=144$ and $n=180$ )

In Figure 4, we show the evolution of the rupture force for two independent sets of experiments. Important general features are similar for all measurement curves of this type: Above a threshold of $I_{\text {crit }} \approx 15 \mathrm{mM}$, a further increasing salt concentration results in a further increase of the adhesion force followed by an apparent saturation. Differences in the qualitative curve shape could be a result of small differences in the interaction between bilayer and support. 

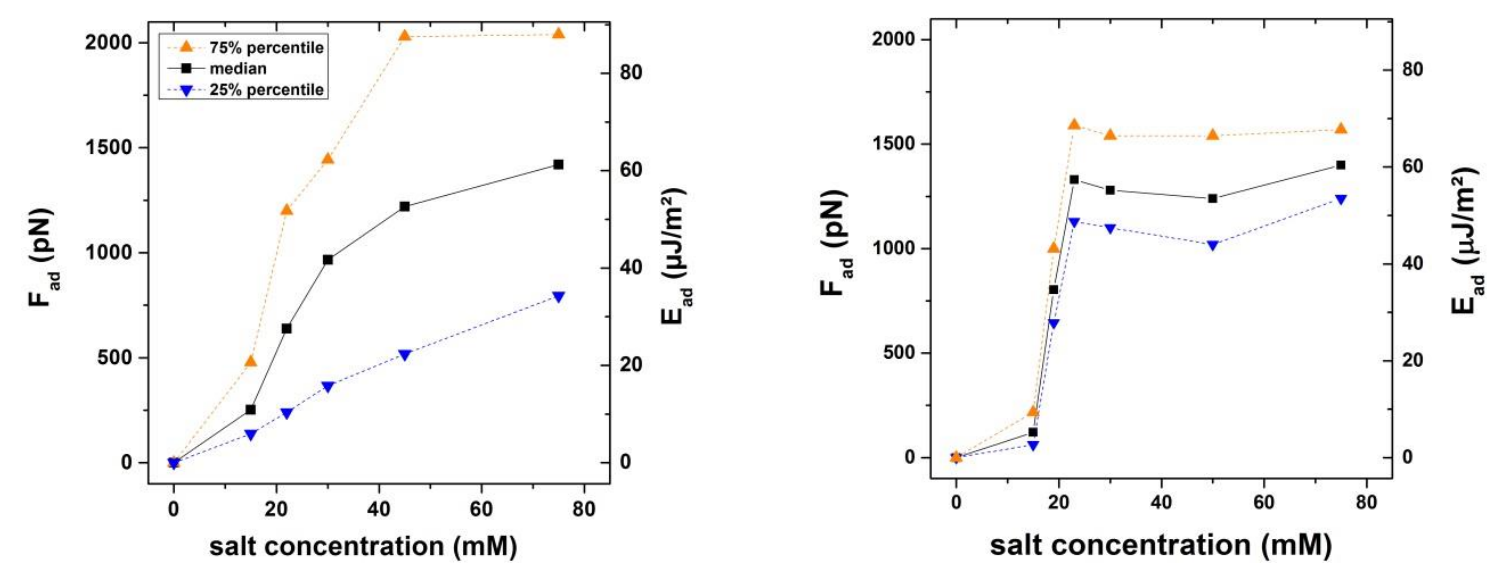

Figure 4. Results of two independent bilayer-silica interaction measurements on mica substrates. The measurements are done using two freshly prepared cantilevers and bilayers. The saturation energy in both cases is about $60 \mu \mathrm{J} / \mathrm{m}^{2}$. Differences in the qualitative curve shape could be a result of small differences in the interaction between bilayer and support. For each salt concentration at least 144 force curves have been evaluated.

From the measured adhesion forces, one can now approximate the adhesion energy, $E_{a d}$, following Derjaguin's approach ${ }^{[24]}$,

$$
E_{\text {ad }}=\frac{F_{\text {add }}}{2 \pi R}(1)
$$

Here $F_{a d}$ represents the adhesion force and $\mathrm{R}$ the radius of the adhering particle. For comparison, we also include this adhesion energy $E_{a d}$ in Figure 4 (right ordinate).

Rentsch et al. ${ }^{[25]}$ and Todd et al. ${ }^{[26]}$ investigated the limits of the Derjaguin approximation and found that the Debye length, $\lambda_{\mathrm{D}}$, is a suitable parameter for checking the validity of the approximation. In our case $\lambda_{\mathrm{D}} \leq 2.5 \mathrm{~nm}$ for a $1: 1$ electrolyte which is much smaller than the radius of our micro particles. Thus, the approximation is clearly valid for our calculations.

To relate these results to nanoparticle-lipid membrane interaction, we complement these results with experiments, where we added silica nanoparticles to a solution of free-standing DOPC vesicles as reported earlier by our group ${ }^{[21]}$. These experiments, as exemplarily shown in Figure 5, show that the endocytosis-like uptake of NPs can be regulated by ion concentration. 

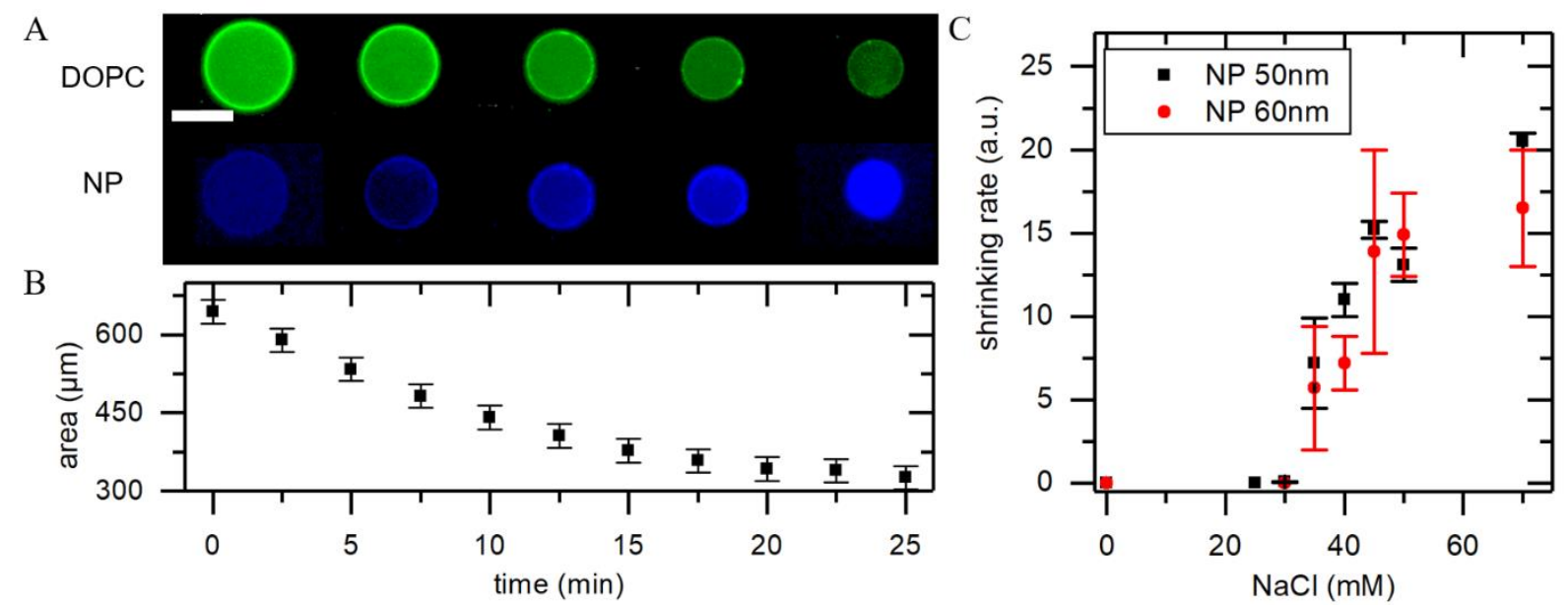

Figure 5. A) Exemplarily shown shrinking of DOPC vesicles (green) in presence of silica NP (blue). B) Surface Area of the vesicle as function of time C) Shrinking rates of DOPC vesicles as function of the $\mathrm{NaCl}$ concentration in presence of $50 \mathrm{~nm}$ and $60 \mathrm{~nm}$ sized NP as a measure for the endocytosis-like NP uptake.

As described in our previous publication, for the endocytosis-like uptake the following basic mechanism can be applied ${ }^{[21]}$ : single adhered NP are engulfed by lipid membrane, subsequently the membrane neck brakes and the engulfed NP is released into the vesicle interior leaving a pore and thus reducing the available membrane area of the vesicle. As long as the pore is open, water can leave the vesicle reducing membrane tension. The membrane coated NP do not leave the vesicle due to the changed surface properties. The resulting decrease in membrane area as shown in Figure 5B is a direct measure for NP uptake. From these measurements shrinking rates are determined and shown in Figure 5C. This uptake process involves the adhesion energy, $E_{\text {adh }}$, the bending energy necessary to engulf the NP with lipid membrane, $E_{b e n}$, and the energy arising from the membrane tension, $E_{\text {ten }}$. According to this theory, a particle uptake is only possible if the adhesion energy is larger than the total membrane energy given by the membrane bending and tension:

$$
E_{\text {adh }} \geq E_{\text {ben }}+E_{\text {ten }}
$$

In the following paragraph in a rough first order approximation we theoretically compare adhesion energy and bending energy. We assume that especially in the beginning of the 
uptake experiment sufficient excess area is available and thus membrane tension is small compared to the bending energy necessary to engulf the NP. Basically, as indicated by the data shown in Figure 5, above a NP size dependent threshold for the ion concentration, higher ion concentrations in the solution induce higher intake rates of the particles. Combining these observations with the theoretical assumptions named above we conclude that the adhesion energy is also increasing, if we assume that other particle and membrane related parameters are constant. Thus, qualitatively the uptake behavior as function of ion concentration is predicted correctly by our model in combination with the adhesion energy determined by AFM experiments.

For quantitative considerations, we now turn to actually calculate the energy contribution for membrane bending and compare it with adhesion energy between silica and DOPC. The bending energy per unit area, $\mathbf{g}_{b}$, according to Helfrich ${ }^{[27]}$ can be simplified to:

$$
\mathrm{g}_{b}=2 k / r^{2}
$$

where $k$ is the bending modulus. With the typical bending modulus for DOPC, $k=10^{-19} \mathrm{~J}$ ${ }^{[28,29]}$, the DOPC bending energies for different nanoparticle sizes $(\mathrm{r}=10 \mathrm{~nm}-70 \mathrm{~nm})$ fall in the range of $2.00 \mathrm{~mJ} / \mathrm{m}^{2}-0.04 \mathrm{~mJ} / \mathrm{m}^{2}$. For the uptake experiments shown in Fig. 5 we obtain from these rough estimations $\mathrm{g}_{b}=0.22 \mathrm{~mJ} / \mathrm{m}^{2}$ Experimentally, we observe a maximum of the adhesion energy of $\mathrm{g}_{b}=0.06 \mathrm{~mJ} / \mathrm{m}^{2}$ as shown in Figure 4. This value already lies in the calculated range for the estimated bending energy, even though the membrane tension is still neglected.

Thus, our AFM experiments can explain the increase in the particle intake qualitatively, but quantitatively the measured values are too small to explain the phenomenon of adhesion driven particle uptake. To elucidate the origin of these quantitative differences, we discuss the role of the asymmetry between vesicle experiments and adhesion measurements using SLB. Toikka et al. investigated the interaction between silica spheres and planar mica surfaces 
without bilayers ${ }^{[30]}$. They used different salt concentrations and found that the repulsion range for $0.1 \mathrm{mM} \mathrm{NaCl}$ is about $50 \mathrm{~nm}$ and for $1 \mathrm{mM} \mathrm{NaCl}$ about $20 \mathrm{~nm}$. Compared to our results as being presented in Figure 2B, the repulsive behavior without salt is very similar to their results for low salt concentrations. From this, we conclude that in the salt free solution the bilayer does not influence the interaction significantly. However, in presence of salt the adhesive behavior is dominated by the bilayer, as the bare silica-mica system does stay repulsive also for higher salt concentrations.

Anderson et al. discussed the interaction between an uncharged bilayer and silica theoretically ${ }^{[23]}$. Basically, there are two theoretical regimes: constant charge and constant potential. In the case of pure water this leads to a purely repulsive electrostatic interaction which is in accordance with our measurements ${ }^{[23,31]}$. For a concentrated salt solution, the system is in the constant potential regime. The dominating interaction force for this case is the double layer force. An approximation of this force gives the Hogg-Healy-Fuerstenau (HHF) equation ${ }^{[32]}$ :

$$
W_{d l}=\frac{\varepsilon_{0} \varepsilon_{r} \kappa\left[2 \psi_{1} \psi_{2}-\left(\psi_{1}^{2}+\psi_{2}^{2}\right) e^{-K \nu}\right]}{e^{K D}-e^{-K D}}
$$

where $\psi_{1,2}$ are the potentials of the surfaces, $D$ is the separation of the surfaces, $k$ is the Boltzmann constant, $\kappa$ is the Debye constant, $\varepsilon_{0}$ is the dielectrical constant and $\varepsilon_{r}$ the dielectric constant of water. For our case, this equation always shows attractive interaction as it is observed in our measurements containing salt in the solution. But the salt concentrations in our experiments are somewhere in between the aforementioned two regimes. The HHF equation can only be first order approximation for our case. Nevertheless, we observe the switching behavior from repulsive to attractive interaction in accordance with ${ }^{[23]}$.

Several other studies also observe significant influence of the specific SLB preparation process on the Bilayer behavior ${ }^{[10,33,34]}$, discussing e.g. also the role of diffusion and domain size. Taking together, the influence of the manufacturing process on SLB is yet not fully 
understood and it has been shown that the preparation process of SLB can greatly influence the measured adhesion forces.

Besides the preparation process there might be a strong influence of the used substrate of the SLB on the determined adhesion energy between bilayer and silica. Tero et al. observed lower diffusion constants on rough surfaces (e.g. glass) than on smooth surfaces (e.g. mica) ${ }^{[12]}$ and Scomparin et al. report about different diffusion constants on mica and glass, using the same bilayer preparation technique ${ }^{[35]}$. In order to investigate the influence of the substrate on our adhesion measurements, we thus repeated our experiments by using of glass slides as a support instead of mica. As depicted in Figure $\mathbf{6}$ for three nominally identical and independent sets of experiments, the measured adhesion forces for DOPC on glass substrates are in average about one order of magnitude larger than on mica but the results seem to indicate no obvious correlation between salt concentration and adhesion forces. Furthermore, all three data sets shown in Figure 6 reveal significant qualitative differences.
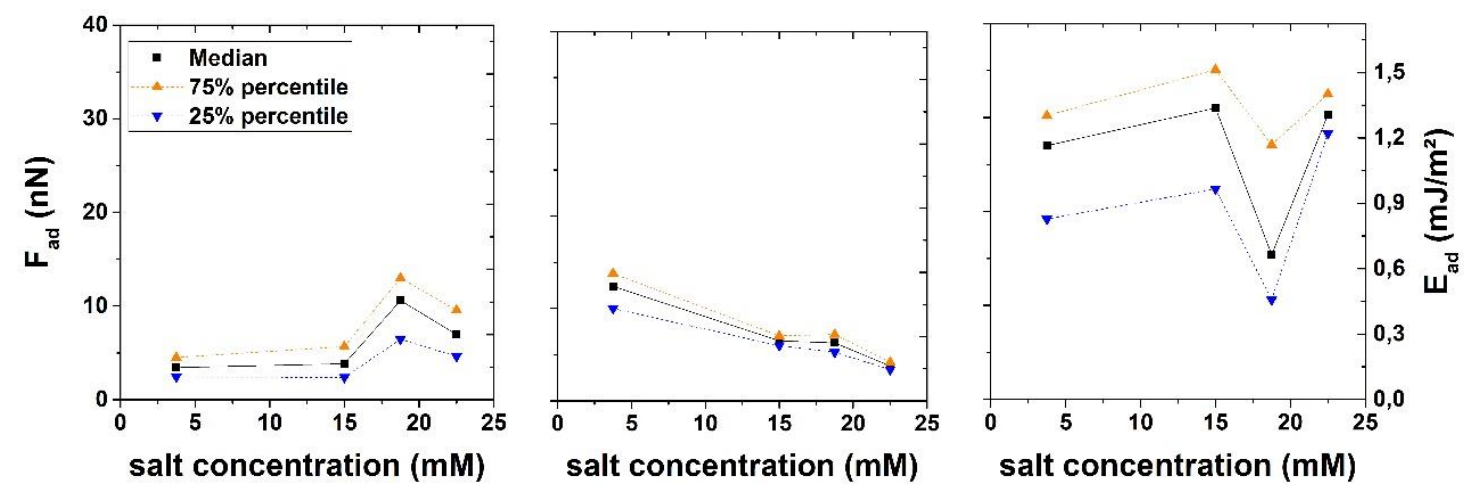

Figure 6. Adhesive forces for bilayer-silica interaction on glass substrates. The independent measurements show no obvious correlation nor a clear dependence on the salt and ion concentration respectively. For each salt concentration at least 167 force curves have been evaluated.entration in presence of $50 \mathrm{~nm}$ and $60 \mathrm{~nm}$ sized NP as a measure for the endocytosis-like NP uptake.

Experiments on glass substrates (at least those used here), surprisingly do not reproduce the qualitative dependency on the salt concentration as shown above for mica. One reason might be the adhesion of the bilayer to the substrate, which might differ for both supports investigated here. For instance, coarse-grained simulations ${ }^{[36]}$ of SLB result in a thin water 
layer of about one nanometer between the substrate and the overlaying bilayer, and the density profiles of lipid and water molecules are calculated for rough surfaces (e.g. glass) and planar surfaces (e.g. mica). In both cases the thin water layers are important, but on smooth surfaces the lipid density is much higher. In other reports it is assumed that there is no water layer between bilayers and glass substrates at all due to ion bridges between bilayer and substrate ${ }^{[33,34]}$. These reports, however, also indicate differences between bilayer adhesion on mica and on glass substrates which is in agreement with our results.

Without a statement about any possible thin interlayers, it seems to be understandable that the energy landscape in the multi-parameter space of SLB adhesion investigations on (rough) glass substrates are much more complex and by far more difficult to control experimentally. What we can state, so far, is that the force setpoint chosen in our experiments using glass supports has a clear influence on the extracted adhesion forces. We show this finding in Figure 7 and Figure 8, where we depict the apparent correlation between setpoint force and adhesion force (Figure 7A) and the one between adhesion probability and setpoint force (Figure 7B). This is not the case for mica supports.

A

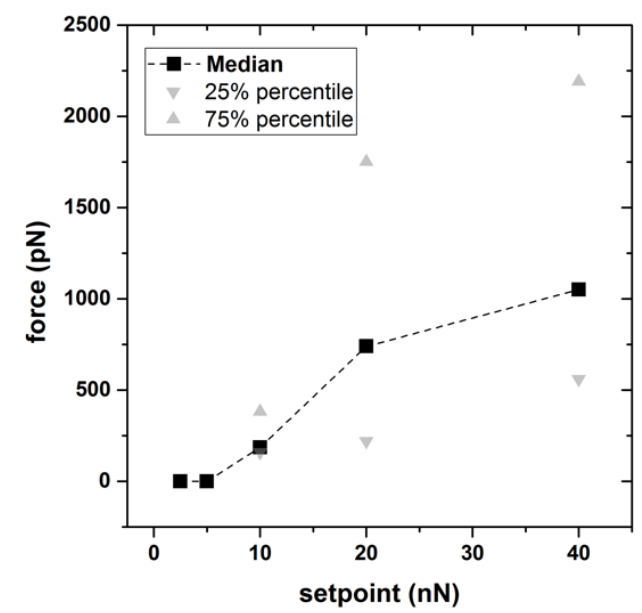

$\mathrm{B}$

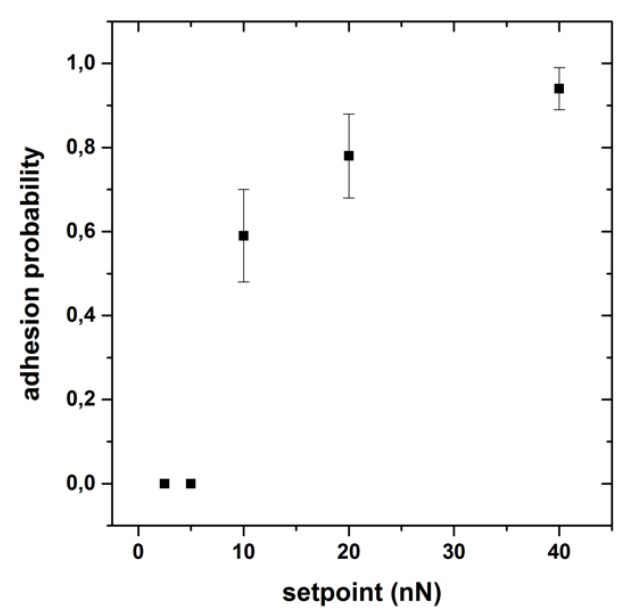

Figure 7. A) Setpoint dependent adhesion force and B) adhesion probability for DOPC bilayers on glass substrate. The pause time is kept constant for these measurements. 
Also the idle time during which the particle is 'pressed' against the bilayer seems to be more important for rough substrates (glass) than for the smooth mica surfaces (see Figure 8).

A

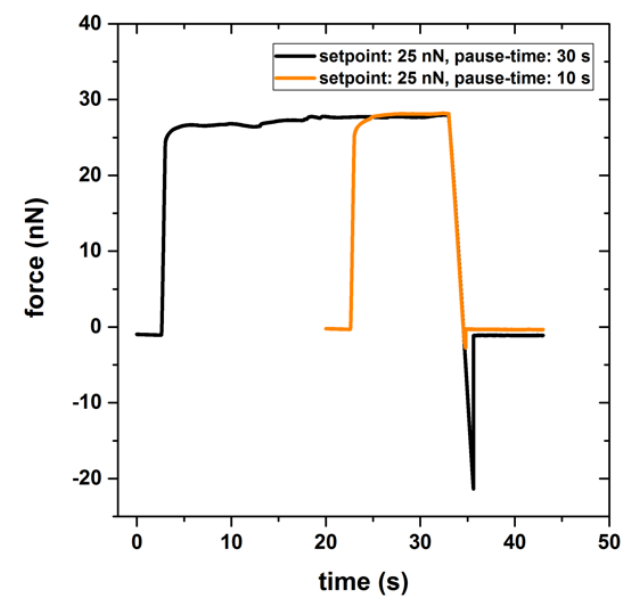

B

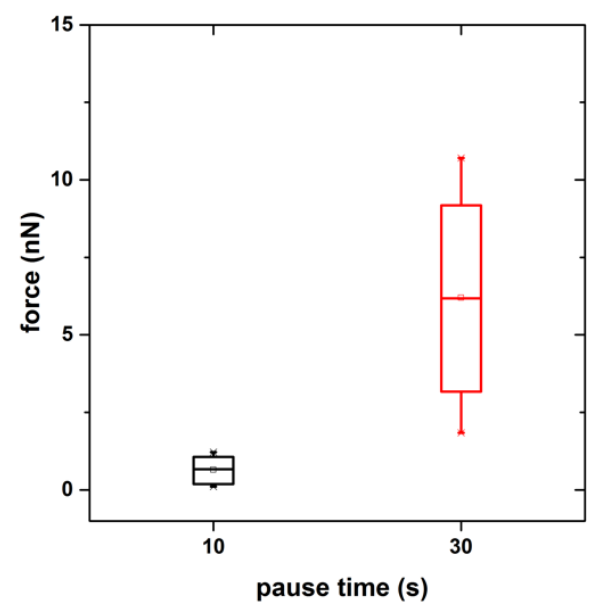

Figure 8. A) force-time curves for two different pause times on glass. It is clearly visible that the adhesion force for $30 \mathrm{~s}$ pause-time is much stronger than for $10 \mathrm{~s}$. B) Boxplot for about 70 curves. The measured adhesion force for a pause time of $30 \mathrm{~s}$ is always greater than the force for a pause time of $10 \mathrm{~s}$.

These findings suggest that in the experimental time scales, kinetic aspects play an important role for the behavior of bilayers on a glass support, in contrast to those on mica.

One possible reason is a more viscous behavior of glass-SLBs which is in accordance with much smaller diffusion constants measured here and in several other studies ${ }^{[3,8]}$. The approaching and contact of the silica bead can be considered as a perturbation of the ionic ordering in the bilayer. As the rearrangement is coupled to the diffusion times, this seems to be on the timescale of our measurements using glass supported SLB. If this is the case, time and force dependent adhesion must be expected.

Another aspect that might explain the strong variations between single measurements are related to geometrical aspects of the surface roughness of the substrate. For a rough substrate, the effective interaction area can vary with the local surface geometry around a specific adhesion site. However, the differences in surface roughness are not very drastic, as we obtain about $R_{\mathrm{q}}=1 \mathrm{~nm}$ for the used glass substrates, while for mica we find $R_{\mathrm{q}} \approx 0.2 \mathrm{~nm}$. Other 
significant differences between the properties of the used substrates here, including the surface charge, chemical makeup or surface silanol coverage might play a significant role as well.

Nevertheless, we see that reported discrepancies in adhesive forces, as well as between experimentally determined and theoretical considerations on nanoparticle intake may originate from a significant influence of the SLB substrate.

\section{Conclusion}

We demonstrate that the ionic strength of the solvent $(\mathrm{NaCl}$ solution in our case) strongly influences the particle-bilayer adhesion as it was expected from particle uptake experiments. For bilayers on mica supports we observe a strong nonlinear increase of the adhesion energy with increasing ion concentration. Our results show that the interaction between a supported lipid bilayer and a silica bead is attractive in the presence of ions in the solution, and repulsive in pure water. This is in agreement with other experimental reports from literature ${ }^{[23]}$ as well as theoretical descriptions ${ }^{[31,32]}$. This finding correlates convincingly with nanoparticle uptake rates in GUV, even though the theoretical model for such an uptake process predicts stronger absolute adhesion values.

Our experimental findings thus show that the adhesion between silica and biological membranes can be regulated by the ionic strength and even be switched. We believe that our results contribute to the understanding of the interaction and the uptake of silica particles by endocytosis. As discussed earlier, the observed effects are likely to play a general role in the interaction between biological membranes and particles, even though the properties of living cells are much more complex and many other effects might mask the effects investigated here. We have also shown that the nature of the support material plays a crucial role for the observed interaction between SLB and silica surfaces. While measurements on glass are difficult to control and to reproduce, smooth mica surfaces are more suitable for gaining 
reproducible results. Thus, it seems not surprising that there are quantitative differences compared to theoretical models describing free-standing bilayers (vesicles).

We conclude, that even SLB experiments can deliver important qualitative data on surface adhesion. However, SLB can for sure not be considered as completely equivalent to freestanding bilayers or even biological membranes. The strong influence of the support material must always be kept in mind and we strongly suggest the combination of different experimental methods like for example the micropipette technique with force spectroscopy on SLB to extract quantitative data on the biologically highly relevant adhesion energy between lipid bilayers and other materials.

\section{Experimental Section}

\section{Vesicle preparation and bilayer formation.}

1,2-Dioleoyl-sn-glycero-3-phosphocholine (DOPC) with a concentration of $25 \mathrm{mg} / \mathrm{ml}$, dissolved in chloroform, was purchased from Avanti Polar Lipids and used without further purification. For fluorescence microscopy, $0.05 \%$ fluorescence dye, DIOC14 from Biotium Inc., was added to the solution. $100 \mu 1$ of the lipid solution was dried by purging with nitrogen gas and placed in a vacuum desiccator for a minimum of 4 hours. Subsequently, the lipid was rehydrated with $15 \mathrm{mM}$ HEPES buffer (4-(2-Hydroxyethyl)piperazine-1-ethanesulfonic acid, Sigma Aldrich)for the mica experiments and with $2.5 \mathrm{mM}$ HEPES for the glass experiments. The mixture was shaken for $30 \mathrm{~min}$ and sonicated in an ultrasound device (Hielscher Ultrasonics, VialTweeter UIS250V) for another $20 \mathrm{~min}$ to produce small unilamellar vesicles (SUV).

Freshly cleaved mica and cleaned glass slides were used as substrate for SLB production. Borosilicate glass slides were purchased from Menzel-Glaeser. To start with a clean and hydrophilic surface, the substrates were additionally treated by an oxygen plasma after thoroughly cleaning with acetone, isopropanol and ultrapure water. AFM experiments were 
carried out with a Nano Wizard from JPK Instruments AG (Berlin, Germany) equipped with a BioCell chamber. The temperature was kept constant at $20^{\circ} \mathrm{C}$ for all experiments. $300 \mu \mathrm{l}$ of the SUV-solution were placed onto the substrate and incubated for $60 \mathrm{~min}$. Afterwards, the solution was gently rinsed with buffer solutions of different salt concentrations. Before and after the experiments, the quality and the properties of bilayer was checked by observing the fluorescence recovery after photobleaching (FRAP) ${ }^{[8]}$. A spot of the fluorescence labeled bilayer was bleached by intense illumination and the subsequent diffusion driven recovery of the bleached spot was observed. This relatively simple experiment nicely reveals the respective diffusion constants, which turn out to be $D_{\text {mica }} \approx 6-8 \mu \mathrm{m}^{2} / \mathrm{s}$ and $D_{\text {glass }} \approx 0.5-1.5$ $\mu \mathrm{m}^{2} / \mathrm{s}$ for 1,2-Dioleoyl-sn-glycero-3-phosphocholine (DOPC) bilayers on the two different substrates. In literature similar experiments result in very comparable diffusion constants ${ }^{[3,8]}$. GUV were prepared by electroformation as described for the first time by Angelova et al. ${ }^{[37]}$. In short, lipids in the desired ratio and $0.05 \mathrm{~mol} \%$ of the fluorescent marker DiOC14 were mixed in chloroform and spread onto flourine tin oxide (FTO)-coated glass slides. The solvent was thoroughly removed through vacuum evaporation. For the swelling procedure, a chamber was assembled from two of the slides and a spacer filled with $150 \mathrm{mM}$ sucrose solution. An AC-voltage was applied for a minimum of 4 hours $\left(f=10 \mathrm{~Hz}, E_{\text {eff }}=0.6 \mathrm{~V} / \mathrm{m}\right)$ at room temperature. The osmolarity of all solutions was measured with an Osmomat 030 (Gonotec, Germany) and adjusted to $150 \mathrm{mM}$.

Monodisperse silica nanoparticles were purchased from nanoComposix (Prague, Czech Republic). Particle size distributions as well as the $\zeta$-potentials were given by the manufacturer: $50 \mathrm{~nm}$ particles: $\mathrm{d}=48.1 \pm 5.3, \zeta=-53.2 \mathrm{Mv} ; 60 \mathrm{~nm}$ particles: $\mathrm{d}=57.8 \pm 3.5 ; \zeta$ $=-44.7 \mathrm{mV}$. 


\section{Cantilever Preparation.}

Silica spheres with a diameter of $7.38 \pm 0.24 \mu \mathrm{m}$ were purchased from microParticles $\mathrm{GmBH}$ (Berlin, Germany) and the tipless cantilevers of type MLCT-O10 from Bruker AFM Probes (Camarillo, USA). Following a technical note from JPK Instruments ${ }^{[38]}$, a UV-hardening glue (BONDIC from VIKO UG, Munich, Germany), was placed in small patches near a bunch of silica spheres on a glass slide. The cantilever was then carefully approached until it dipped into such a glue patch and taken up a small drop. This glue covered cantilever was then carefully pressed onto one of the silica spheres. The glue was then cured by UV illumination minimum of one hour to guarantee full polymerization. The spring constant $k$ of the cantilevers was $k=0.03 \mathrm{~N} / \mathrm{m}$ for those for mica substrates and $k=0.1 \mathrm{~N} / \mathrm{m}$ for the ones for the glass substrate experiments.

Table 1 shows the settings of the AFM used for the adhesion measurements.

Table 1. Force spectroscopy settings

\begin{tabular}{lcc}
\hline & Mica & Glass \\
\hline $\begin{array}{l}\text { approach } \\
\text { speed }\end{array}$ & $0.5 \mu \mathrm{m} \mathrm{s}-1$ & $0.5 \mu \mathrm{m} \mathrm{s}-1$ ) \\
$\begin{array}{l}\text { force } \\
\text { setpoint }\end{array}$ & $26 \mathrm{nN}$ & $80 \mathrm{nN}$ \\
$\begin{array}{l}\text { pause time } \\
\text { (constant } \\
\text { height) }\end{array}$ & $0 \mathrm{~s}$ & $5 \mathrm{~s}$ \\
$\begin{array}{l}\text { retraction } \\
\text { speed }\end{array}$ & $0.21 \mu \mathrm{m} \mathrm{s}-1$ & $0.21 \mu \mathrm{m} \mathrm{s}-1$ \\
\hline
\end{tabular}

\section{Statistical Analysis}

1. Preprocessing: All force curves where processed with the following established steps: base line determination, contact point determination at the intersection of base line and linear fit to the repulsive region of the force curve. Less than $10 \%$ of the force curves where sorted out if they contained two or more ruptures. 2. Data presentation: If not explicitly declared as median and percentile the shown values are mean values and standard \pm deviation. 3. Sample sizes are 
given in each caption where applicable. 4. No statistical tests and according software were used.

\section{Acknowledgements}

This work has been sponsored by the cluster of excellence „Nanosystems Initiative Munich, NIM“. Moreover, we thank Dominik Breyer for enlightening discussions and technical assistance.

\section{References}

[1] E. Gorter, F. Grendel, J. Exp. Med. 1925, 41, 439.

[2] E. Sackmann, Science (80-. ). 1996, 271, 43.

[3] L. K. Tamm, H. M. McConnell, Biophys. J. 1985, 47, 105.

[4] E. T. Castellana, P. S. Cremer, Surf. Sci. Rep. 2006, 61, 429.

[5] R. P. Richter, A. R. Brisson, Biophys. J. 2005, 88, 3422.

[6] S. Mornet, O. Lambert, E. Duguet, A. Brisson, Nano Lett. 2005, 5, 281.

[7] T. Cha, A. Guo, X. Zhu, Biophys. J. 2006, 90, 1270.

[8] K. J. Seu, A. P. Pandey, F. Haque, E. A. Proctor, A. E. Ribbe, J. S. Hovis, Biophys. J. 2007, 92, 2445.

[9] E. Kalb, S. Frey, L. K. Tamm, Biochim. Biophys. Acta-Biomembr. 1992, 1103, 307.

[10] T. E. Starr, N. L. Thompson, Langmuir 2000, 16, 10301.

[11] M. Tanaka, E. Sackmann, Nature 2005, 437, 656.

[12] R. Tero, Materials (Basel). 2012, 5, 2658.

[13] R. A. Petros, J. M. DeSimone, Nat Rev Drug Discov 2010, 9, 615.

[14] B. D. Plouffe, D. N. Njoka, J. Harris, J. Liao, N. K. Horick, M. Radisic, S. K. Murthy, Langmuir 2007, 23, 5050.

[15] A. Verma, O. Uzun, Y. Hu, Y. Hu, H. Han, N. Watson, S. Chen, D. J. Irvine, F. Stellacci, Nat. Mater. 2008, 7, 588.

[16] T. Wang, J. Bai, X. Jiang, G. U. Nienhaus, ACS Nano 2012, 6, 1251.

[17] I. Koltover, J. O. Rädler, C. R. Safinya, Phys. Rev. Lett. 1999, 82, 1991.

[18] A. H. Bahrami, R. Lipowsky, T. R. Weikl, Phys. Rev. Lett. 2012, 109, 188102.

[19] B. G. Trewyn, S. Giri, I. I. Slowing, V. S.-Y. Lin, Chem. Commun. 2007, 31, 3236.

[20] C. Westerhausen, F. G. Strobl, R. Herrmann, A. T. Bauer, S. W. Schneider, A. Reller, A. Wixforth, M. F. Schneider, Biophys. J. 2012, 102, 1032.

[21] F. G. Strobl, F. Seitz, C. Westerhausen, A. Reller, A. a Torrano, C. Bräuchle, A. Wixforth, M. F. Schneider, Beilstein J. Nanotechnol. 2014, 5, 2468. 
[22] E. Rascol, J.-M. Devoisselle, J. Chopineau, Nanoscale 2016, 8, 4780.

[23] T. H. Anderson, Y. Min, K. L. Weirich, H. Zeng, D. Fygenson, J. N. Israelachvili, Langmuir 2009, 25, 6997.

[24] B. Derjaguin, Kolloid-Zeitschrift 1934, 69, 155.

[25] S. Rentsch, R. Pericet-Camara, G. Papastavrou, M. Borkovec, Phys. Chem. Chem. Phys. 2006, 8, 2531.

[26] B. A. Todd, S. J. Eppell, Langmuir 2004, 20, 4892.

[27] W. Helfrich, Zeitschrift für Naturforsch. C 1973, 28, 693.

[28] Z. Chen, R. P. Rand, Biophys. J. 1997, 73, 267.

[29] J. F. Nagle, M. S. Jablin, S. Tristram-Nagle, K. Akabori, Chem. Phys. Lipids 2015, 185, 3.

[30] G. Toikka, R. Hayes, J. Colloid Interface Sci. 1997, 191, 102.

[31] J. Gregory, J. Colloid Interface Sci. 1975, 51, 44.

[32] R. Hogg, T. W. Healy, D. W. Fuerstenau, Trans. Faraday Soc. 1966, 62, 1638.

[33] H. M. McConnell, L. K. Tamm, R. M. Weis, Proc. Natl. Acad. Sci. U. S. A. 1984, 81, 3249.

[34] R. Merkel, E. Sackmann, E. Evans, J. Phys. 1989, 50, 1535.

[35] C. Scomparin, S. Lecuyer, M. Ferreira, T. Charitat, B. Tinland, Eur. Phys. J. E 2009, $28,211$.

[36] C. Xing, R. Faller, J. Phys. Chem. B 2008, 112, 7086.

[37] M. I. Angelova, S. Soleau, P. Meleard, J. F. Faucon, P. Bothorel, Prog Colloid Polym Sci 1992, 89, 127.

[38] Attaching Microspheres to Cantilevers Using the NanoWizard ${ }^{\circledR}$ Life Science Stage and AFM Head Microsphere Cantilever Tips, n.d. 
The effect of ionic strength on the adhesion between silica and phospholipid membranes is shown. On mica supports at low ionic strength the interaction is repulsive, becomes attractive above a threshold, rapidly increases with increasing ionic strength and saturates. The same holds for uptake rates of silica nanoparticles in vesicles. However, on glass the adhesion does not follow this trend.

\section{Specific adhesion energy}

C. G. Wittmann, A. Kamenac, F. G. Strobl, D. Czubak, A. Wixforth, C. Westerhausen*

Ionic strength and the supporting material strongly influence the adhesion of silica to supported lipid bilayers

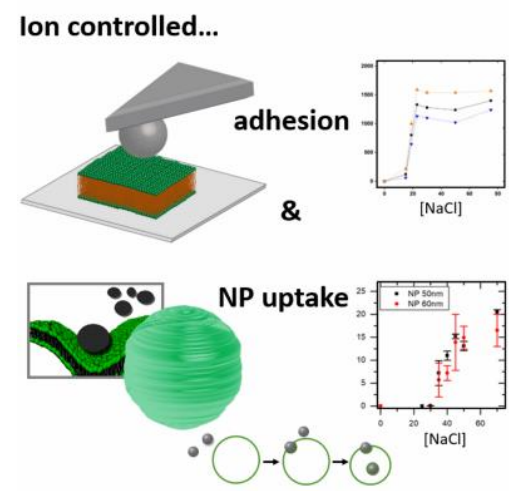

ToC figure 